\title{
FACILE SYNTHESIS OF GRAPHENE VIA SULFUR INTERCALATION AND THERMAL EXFOLIATION AND ITS APPLICATION IN ELECTROCHEMICAL SENSING OF L-TRYPTOPHAN
}

\author{
V. Sivasankar ${ }^{1}$, E. Senthil Kumar ${ }^{1}$ and R. Suresh Babu ${ }^{2}$ \\ S. Raghu ${ }^{2}$ and R. A. Kalaivani ${ }^{1, *}$ \\ ${ }^{1}$ Department of Chemistry, Vels University, Chennai-600 117, Tamil Nadu, India \\ ${ }^{2}$ Department of Vels Advanced Energy Research, Vels University, \\ Chennai-600 117, Tamil Nadu, India. \\ *E-mail: rakvani@yahoo.co.in
}

\begin{abstract}
An suitable along with an economical production move toward was projected intended for the synthesis of graphene from thermally expanded graphite in a simple and scalable method by sulfur atom intercalation to tailor its properties. Thermally expanded graphite was further subjected to modified Hummer's method and thermally treated to exfoliate graphene (TEG). To detect the sensitivity of L-tryptophan (L-Trp, the amperometric reaction of the TEG adapted glassy carbon electrode (TEG/GCE) exist employed. The TEG/GCE modified electrode behaviors of L-Trp were carefully examined by cyclic voltammetry in the concentration variety of $13 \mu \mathrm{M}$ to $1 \mathrm{mM}$ with a detection limit of $4.3 \mu \mathrm{M}(\mathrm{S} / \mathrm{N}=3)$. L-Trp showed enhancement in the oxidation peak existing through a negative shift oxidation $(+0.53 \mathrm{~V})$ compared through that on the bare GCE $(+0.75 \mathrm{~V})$. Thus, the promising TEG/GCE modified electrode showed that the sensor exhibited high selectivity, reproducibility, and good long-term stability. This modified electrode was also fairly effective for the determination of L-Trp in milk samples.
\end{abstract}

Keywords: Thermally expanded graphene, intercalation, L-Tryptophan, cyclic voltammetry.

(C) RASĀYAN. All rights reserved

\section{INTRODUCTION}

Graphene represents one member of an increasingly populated class of carbon allotropes. ${ }^{1}$ The investigation of the physical properties of graphene has lead to a large number of groundbreaking discoveries. ${ }^{2}$ Thus, graphene enclose rising character like areas of inspect attracting massive interest into scholarly globe as well as industry. ${ }^{3-5}$ Scalable, commercial growth is a key requirement to build employ of the best properties of mono- and few-layer grapheme. ${ }^{6}$ On the origin of its new properties such as fundamentally greater electrical conductivity, high specific surface area, wonderful mechanical strength, and unusual thermal conductivity, graphene has been viewed unit promising materials in the fields of electronics and Photonics, biosensor and catalysis, graphene-based batteries, supercapacitors, etc. ${ }^{7}$ The exfoliation way of producing graphene sheets has been invent to be regular and trouble-free, here addition to has concerned the direct reflection of the scientific community. ${ }^{8-10}$ Now growth, graphene has been planned to be promising and convenient electrode modified material for sensors due to its straightforward chemical modification with other groups and molecules; it has also been reflect on wonderful material for electrochemistry in present years. ${ }^{11}$

One of the amino acid necessary used for protein biosynthesis, nitrogen balance, muscle accumulation preservation and body influence in human is L-Tryptophan (L-Trp). ${ }^{12}{ }^{13}$ Hence, express, trouble-free, perceptive low detection performance is important for the amino acid. Electrochemical techniques are employed used for superior sensitivity, accuracy and uncomplicated operation manner. By using L-Trp at 
the typical electrodes poor electron transfer and high over potential were resulted. So, chemically modified electrodes (CMEs) is used to get enhanced sensitivity and decrease the over potential used for electrochemical oxidation of the above mentioned amino acid. ${ }^{14}$

In this article, the realization of graphene synthesis depends on the safe hands and non-hazardous methods, and large-scale make up at small charge. In this outlook, the travel toward that crucial lead to the partition of graphene among thermal exfoliation to be studied. There are few reasons for the selection of thermal exfoliation method. The thermal exfoliation of graphene, intercalated sulfur atom at a faster rate. The graphite expands to hundreds of times and outcome in enlarged objects with reduce the thickness and enhance in resistance temperature. This development expression as the exfoliation of graphite flakes. The obtained thermally exfoliated graphene was immobilized with glassy carbon electrode (TEG/GCE) was a determination of L-Trp and its electrochemical arrangement of L-Trp measured at working electrode and compared during the bare GC electrode. The result indicated a good electrocatalytic ability towards the oxidation of L-Trp by using TEG/GCE, it exhibited an excellent determination, performance shows the good linear response of L-Trp concentration from $13 \mu \mathrm{M}$ to $1 \mathrm{mM}$ with a detection limit of $0.4 \mu \mathrm{M}(\mathrm{S} / \mathrm{N}=$ $3)$.

\section{Reagents and Instruments}

\section{EXPERIMENITAL}

Chemical reagents such as Graphite flakes $350 \mu \mathrm{M}$, potassium permanganate $\left(\mathrm{KMnO}_{4}\right)$, sulfuric acid $\left(\mathrm{H}_{2} \mathrm{SO}_{4}\right)$, hydrochloric acid $(\mathrm{HCl})$, hydrogen peroxide $\left(\mathrm{H}_{2} \mathrm{O}_{2}\right)$, potassium chloride $(\mathrm{KCl}) \mathrm{N}$, N-methyl pyrrolidine (NMP) were obtained from MERCK. A stock solution of $(0.001 \mathrm{M})$ L-Tryptophan was prepared in dilute alkali solution. 0.1 M phosphate buffer saline (PBS) was used as supporting electrolyte, which was prepared by the count stock solution of $0.1 \mathrm{M} \mathrm{KH}_{2} \mathrm{PO}_{4}$ and $0.1 \mathrm{M} \mathrm{K}_{2} \mathrm{HPO}_{4}$ and adjusting the (pH $6.5-7.0$ ) with $0.1 \mathrm{M} \mathrm{NaOH}$. All the supplementary chemical were of analytical grade. Double distilled water was used throughout all the studies.

X-ray powder diffraction study of the synthesized samples was performed among Bruker AXS D8 Advance $\mathrm{X}$-ray diffract meter. The D8 Advance system was equipped with a $2.2 \mathrm{~kW} \mathrm{Cu}$ anode X-ray tube and a LynxEye position sensitive detector. $\mathrm{Cu}$ K $\alpha$ radiation $(\lambda=1.5406 \AA)$ was used to obtain all powder patterns. Scanning Electron Microscope (SEM) was employed for the determining the morphology and elemental composition. SEM was operated by Hitachi S-3400 N. High- resolution transmission electron microscopy (HR-TEM) FEI, TECHNAI G2 $300 \mathrm{kV}$. Electrochemical studied were performed an electrochemical cell at room temperature in the course of three-electrode system pattern. The auxiliary electrode used is platinum electrode and saturated calomel electrode is employed as the reference electrode. Here CHI 760D electrochemical workstation, (CH Instrument, USA) was occupied for all the electrochemical measurements.

\section{Thermal Exfoliation of Graphite}

The method to make expanded graphite flakes is followed as Natural graphite powder $5 \mathrm{~g}$ and thiourea 10 $\mathrm{g}(1: 2)$ was taken in a silica crucible, in this mixture was added $20 \mathrm{~mL}$ of deionized water was thoroughly added and mixed with a glass rod. The obtained paste-like the mixture is kept in a muffle furnace for $6 \mathrm{~h}$ at $550{ }^{\circ} \mathrm{C}$, further, sulfur intercalated graphite powder was grinded and used to synthesize graphene oxide.

\section{Reduction of Graphene Oxide}

Graphene oxide was synthesized by using improved Hummer's method. ${ }^{15} \mathrm{GO}(500 \mathrm{mg})$ was added to water $(200 \mathrm{ml})$ and results into uniform brown distribution. Its afterward ultrasonicated via ultrasonic bath cleaner awaiting it becomes clear. $2 \mathrm{ml}$ of hydrazine hydrate was then further and heated to $100^{\circ} \mathrm{C}$ under ice-cold condition for about 24 hours. Therefore reduced GO gradually precipitated exposed as a black solid. Afterward it was washed and filtered with water and methanol. It was then dehydrated under a regular air flow during the solid product. ${ }^{16}$ The obtained reduced graphene oxide was further thermally exfoliated and purified at $550{ }^{\circ} \mathrm{C}$ for $30 \mathrm{~min}$, and then the desired graphene sheets were obtained. 


\section{Fabrication of TEG Modified Electrode}

The GCE surfaces were superior by polishing through $0.5 \mu \mathrm{m}$ alumina powder with the polishing stuffing to make a mirror polished surface and washed with deionized (DI) water and dried. Immobilization of the TEG onto the working electrode (GC) was performed by first preparing a suspension of the TEG along with the concentration of $2 \mathrm{mg}$ in $5 \mathrm{~mL}$ of NMP with $10 \mathrm{~min}$ sonication. The complete dispersed of the suspension $(5 \mu \mathrm{L})$ was then added by drop casted method onto the electrode surface. The coating was then allowed to dry for $5 \mathrm{~h}$ at room temperature, the NMP solvent was completely evaporated and it was washed with DI water to remove the unbound materials remaining on to the electrode surface.

\section{Milk Sample Preparation}

The real sample analysis of L-Trp encloses two different milk samples has been bring from local shops. It was then investigated with TEG modified electrode a similar procedure as reported earlier ${ }^{14}$ in brief, $10 \mathrm{~mL}$ of the boiled milk and filtered then diluted to 10 times. To $2 \mathrm{~mL}$ of diluted milk sample, the standard L-Trp solution was promote and the standard L-Trp spiked milk sample was examine to analysis.

\section{Formation Mechanism of TEG}

\section{RESULT AND DISCUSSION}

Since exactly exfoliation is a phase exchange which occurs on well-defined high temperature heating is necessary for excited and the bulk conventional thermal groundwork is a flame in which the intercalated graphite is transported. ${ }^{17}$ The flame is positioned in a furnace, which can be lined with $\mathrm{SiO}_{2}-\mathrm{Al}_{2} \mathrm{O}_{3}$ material to improve the test. ${ }^{18}$ The exfoliation is small when the graphite particle size is moreover small; the least quantity of particle size is $>75 \mu \mathrm{m} .{ }^{19}$ The experimental setup was illustrated by thermally exfoliated graphite to graphene as shown in Fig.-1. The typical nature graphite flakes and thiourea were mixed with silica crucible. When trust the mixture in a muffle furnace for $550^{\circ} \mathrm{C}$ at $6 \mathrm{~h}$. The possible mechanism of thermally exfoliated graphite as following steps:

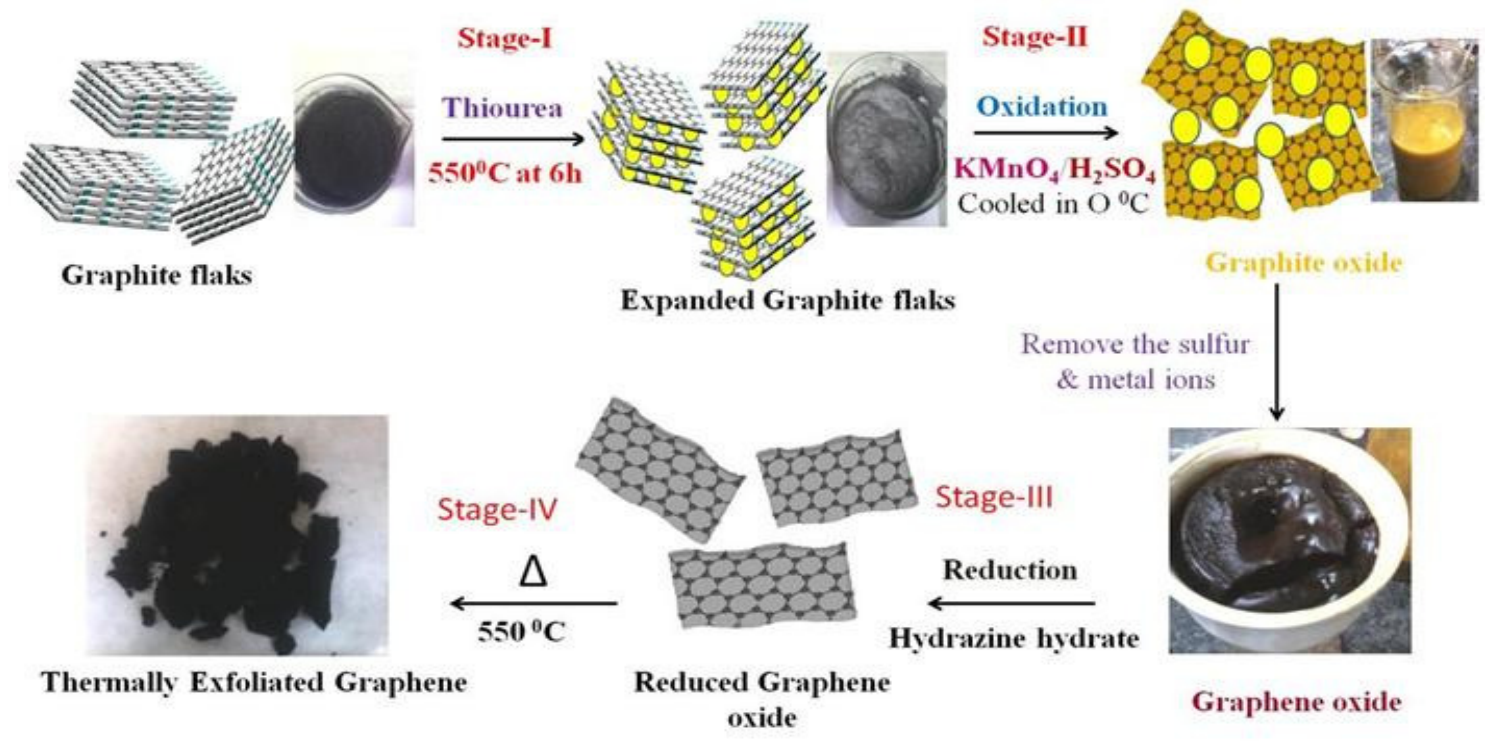

Fig.-1: Thermally exfoliated graphite to grapheme

(i) The initial exfoliation of graphite with thiourea, act as fuel combustion as well as the intercalated agent of sulfur atom to a fast breaking of the weak van-der-waals forces in graphite multi staking layer at $550{ }^{\circ} \mathrm{C}$. Graphite flakes expanded to sulfur intercalated graphite, the inter layers distance in high temperature was increased.

(ii) Exfoliated graphite was oxidized graphite gives graphite oxide, where in graphite layers are separated by sulfur and other oxygen-containing groups like epoxy (-O-), hydroxyl (-OH), carboxyl (-COOH). 
(iii) When the graphene oxide chemically reduced to the surface of graphene oxide to first eliminated weak oxygen group epoxy (-O-), and other oxygen groups.

(iv) Chemically reduce graphenes bear a small amount of oxygen groups on their surface of reduced graphene to complete eliminated during the thermally exfoliated process to obtained thermally exfoliated graphene was further confirmed by XRD, Raman spectrum, FESEM, HRTEM and electrochemical analysis.

\section{XRD, Raman, FESEM and TEM Characterization}

The X-ray diffraction tool to estimate the efficiency of direction monitoring the crystal structure Fig.-2A. shown the thermally exfoliated graphite to graphene oxide (Brown line) synthesis to complete the purification of graphene(Yellow line) by oxidative and reductive process having ions like $\mathrm{K}, \mathrm{Mn}$, $\mathrm{S}$, in this case for removal of all ions to further characterized XRD pattern to shows the observed broad peak at $25.56^{\circ}$ corresponding to the (002) hexagonal plane of graphene as shown Fig.-2A. The d-spacing value at $0.34 \mathrm{~nm}$ to confirm the hexagonal crystalline nature of graphite carbon and the small peak at value of $2 \theta$ at $42.9^{\circ}$ corresponding to the 100 reflections, respectively. Fig. 2B showed Raman scattering is great approachable to the electronic structure and proves to be an imperative tool for determining the density of defect in graphene sheets. In some cases, the Raman spectrum of graphene is characterized by two foremost features, the $\mathrm{G}$ mode arising from the first order scattering of the $\mathrm{E}_{2} \mathrm{~g}$ phonon of $\mathrm{sp}^{2} \mathrm{C}$ atoms which can be used to explain the degree of graphitization and the signal display within raman spectrum of the TEG showed prominent G-band at $1587 \mathrm{~cm}^{-1}$ and the $\mathrm{D}$ mode arising from a breathing mode of k-point photons of $\mathrm{A}_{1} \mathrm{~g}$ symmetry prominent signal at $1350 \mathrm{~cm}^{-1} .{ }^{20,21}$
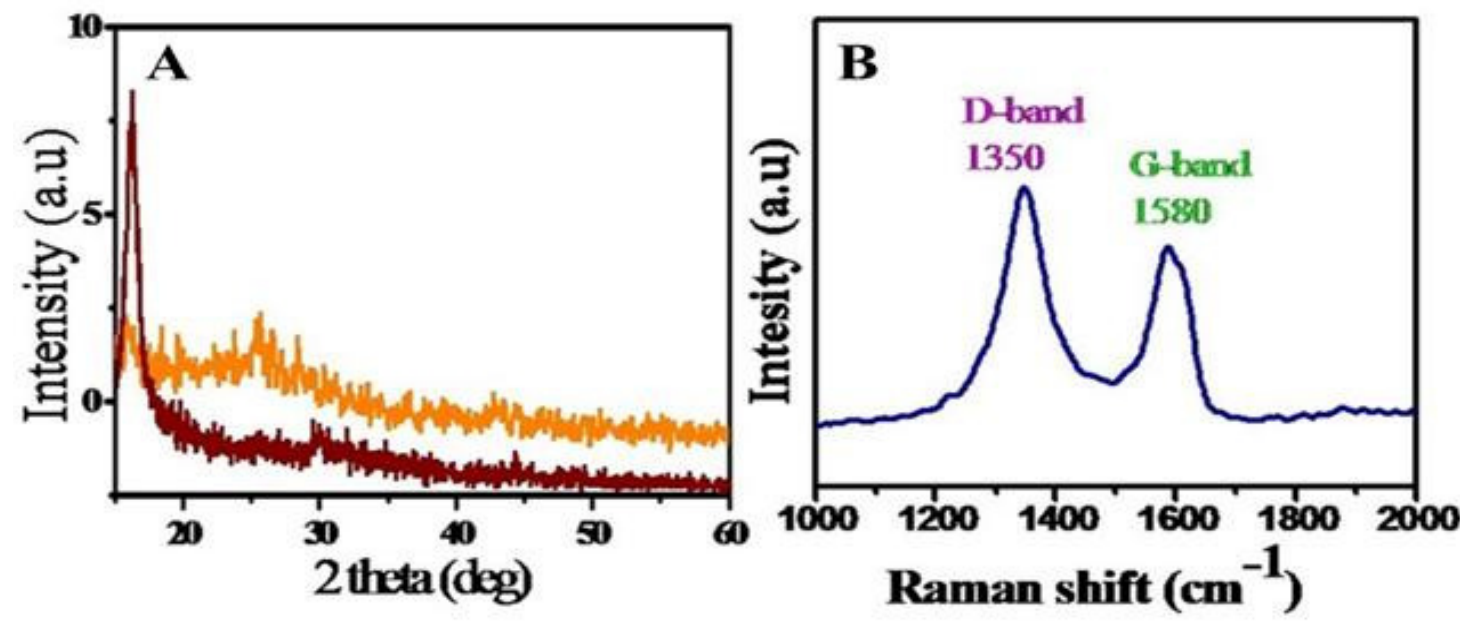

Fig.-2: (A) XRD pattern of graphene oxide and reduced graphene oxide, (B) Raman spectrum of reduced graphene oxide.

The Intensity ratio between $\mathrm{D}$ and G-band ( $\mathrm{I}_{\mathrm{D}} / \mathrm{I}_{\mathrm{G}}$ ratio) used since an indication the degree of disorder in a carbon structure to found value at 1.36 due to the generation on the surface of TEG was found to be no more oxygen-containing groups during the thermal exfoliation process. This suggests to the thermally exfoliated graphene sheets are more disordered, which is consistent with the corrugation and scrolling arrangement observed by FE-SEM and HR-TEM. The face morphological analysis of the TEG/GCE modified electrode was experimental by FE-SEM as shown in Fig.-3A. The sample is clearly seen in folded sheets of few-layered graphene as prepared thermal expansion of graphite flakes by using thiourea as a initial fuel for graphite expansion and further increase the temperature, the graphite to exfoliated graphite flakes and its further used by oxidation and reduction process to be form few layer and large surface of graphene. HR-TEM image shows structural information of TEG synthesized from graphite flakes. It's clearly seen that the transparent graphene sheets folded structure indicates the presence of few layers 
graphene of a honeycomb structure and large particle size $(500 \mathrm{~nm})$ a highly porous nature of graphene synthesized by the thermal exfoliation method as shown in Fig. 3B. A hexagonal ring-like summarized consisting diffraction spots for each assemble of diffraction was results with selected electron diffraction (SAED). The hexagonal pattern with few layers of graphene sheets is highly crystalline in nature and it has been indicated in the ring-like spot.(inset of Fig.-3B).

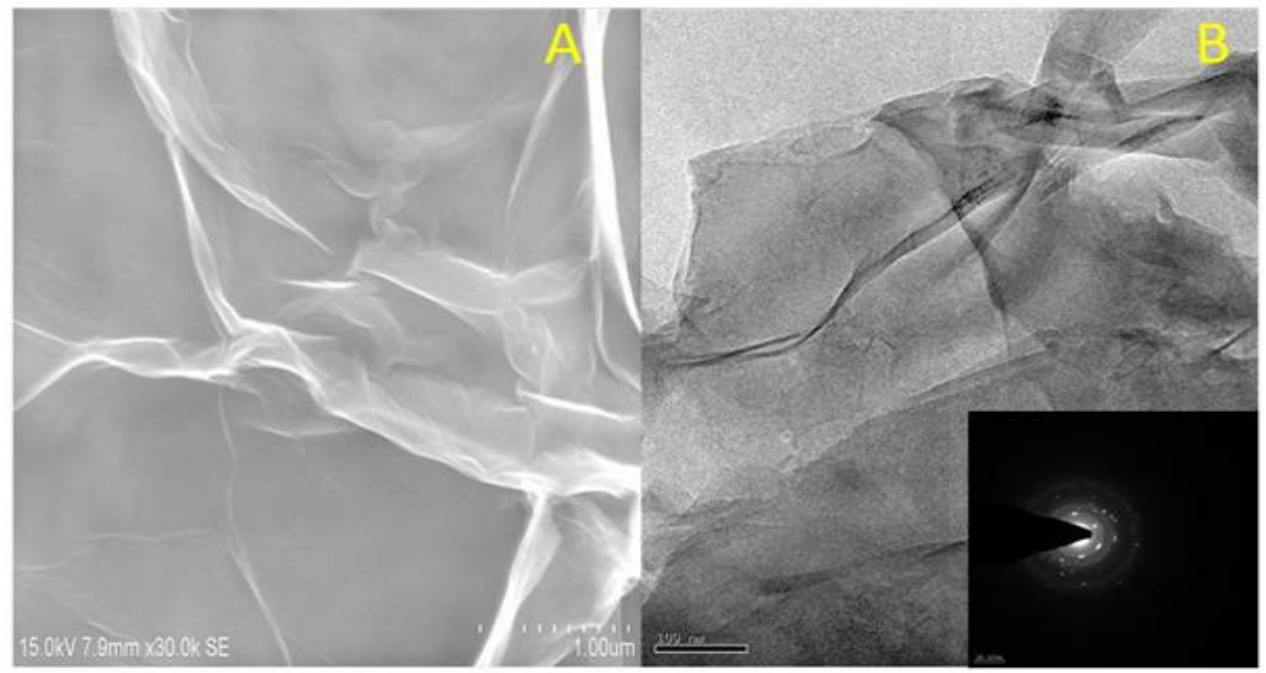

Fig.-3: (A) FESEM and (B) TEM image of thermally exfoliate graphene (insert into SAED pattern)

\section{Electrochemical Characterization of TEG/GCE}

Figure-4A shows the electrochemical behavior of bare GC electrode (a) and TEG/GCE (b) in $10 \mathrm{mM} \mathrm{KCl}$ with $5 \mathrm{mM}$ of $\mathrm{K}_{3}\left[\mathrm{Fe}(\mathrm{CN})_{6}\right]$ at $50 \mathrm{mV} \mathrm{S}^{-1}$. When there is redox peak was obtained in a bare electrode and modified electrode in the presence of redox probe obtained at $\left(\Delta \mathrm{E}_{\mathrm{pa}}\right) 0.38 \mathrm{~V}$ and $0.26 \mathrm{~V}$ consequent. After TEG was modified the GC surface, the electrochemical piece of the $\left[\mathrm{Fe}(\mathrm{CN})_{6}\right]$ was extensively improved the peak current and smaller peak potential due to the high conductivity of TEG. Electrochemical impedance spectroscopy (EIS) is an efficient presentation to study the margin properties of surface modified electrodes. The electron transfer resistance (Ret) of the electrode surface corresponds to the half circle diameter of the Nyquist diagram and can be used to express the interface characteristics of the electrode. Fig. 4B showed the outcome for the Nyquist plots on bare GCE and TEG/GCE. For the bare GCE, the Ret was $4953 \Omega$. After modifying TEG, the Ret $(88 \Omega$ ), which was reflected by the look of extensively decreased in the half circle part of the spectrum. The results predicted that the TEG was sufficiently immobilized on the GCE surface; the TEG gained an excellent conductive material and accelerated electron transfer at the electrode surface.

\section{Electrocatalytic Oxidation of L-Trp at TEG/GCE}

Now order to expected the electrocatatytic behavior of the TEG/GCE toward the oxidation of L-Trp was obtained within comparison with bare GCE by cyclic voltammetry in $0.1 \mathrm{M}$ phosphate buffer saline $(\mathrm{pH}$ 7.0) at scan rate of $50 \mathrm{mV} \mathrm{S}^{-1}$. When there is negligible oxidation peak was obtained from bare GCE at about $0.75 \mathrm{~V}$ (Fig. 5A curve b). Interestingly, the TEG modified electrode shifted oxidation peak potential at $0.55 \mathrm{~V}$ (curve a) with a severe increase in current was obtained as shown in Fig.-5A. The decreases of oxidation over potential and increase the peak current indicates TEG/GCE has greater electrocatalytic activity towards L-Trp oxidation.

Fig.-5B shows the various concentration of L-Trp was investigated by the cyclic voltammetry using modified TEG/GCE was examined. The L-Trp oxidized peak potential was observed positively increase peak of $0.57 \mathrm{~V}$ to $0.65 \mathrm{~V}$ vs SCE. The insert of Fig.-5B shows the corresponding calibration plot of catalytic current response versus concentration of $13 \mu \mathrm{M}$ to $1 \mathrm{mM}$ L-Trp was observed that the modification of 
RASĀYAN $J$. Chem.

Vol. 10 | No. 4 |1232-1241 | October - December | 2017

TEG/GCE exhibits a very linear response of correlation coefficient $\left(\mathrm{R}^{2}=0.9961\right)$. The detection limit of the L-Trp were $4.3 \mu \mathrm{M}(\mathrm{S} / \mathrm{N}=3)$ along with a sensitivity of $0.1651 \mu \mathrm{A} / \mu \mathrm{M}$.

\section{Amperometric Determination of L-trp at TEG/GCE}

Initially, in order to optimize the prepared potential, the hope of the L-Trp response to the applied potential to be calculated by the hydrodynamic voltammetry. The electrocatalytic activity of the TEG/GCE permitted the suitable detection of L-Trp at minor potentials among high sensitivity. In the existence of L-Trp, the current improve response from $+0.55 \mathrm{~V}$, receiving a maximum value of $+0.60 \mathrm{~V}$ with a sigmoidal shape (Fig. not shown). Here compare, the bare GCE showed the detection of L-Trp only at very higher potentials with reduced the sensitivity. To be clear that the TEG have contributed the enhanced of the peak current for the electrocatalytic oxidation of L-Trp. Hence, a potential of $+0.60 \mathrm{~V}$ was applied during amperometric studies. Figure- 6 shows the quality steady-state catalytic current-time reaction of the TEG/GCE with a following injection of L-Trp in the dynamic condition in phosphate buffer saline solution, at a fixed potential of $+0.60 \mathrm{~V}$. As shown through the consecutive additions of $30 \mu \mathrm{M} \mathrm{L}-\operatorname{Trp}$ in $0.1 \mathrm{M}$ PBS (pH 7.0). It was observed that the TEG/GCE responded so quickly to the substrate that it could obtain approximately $90 \%$ of response within $5 \mathrm{~s}$.
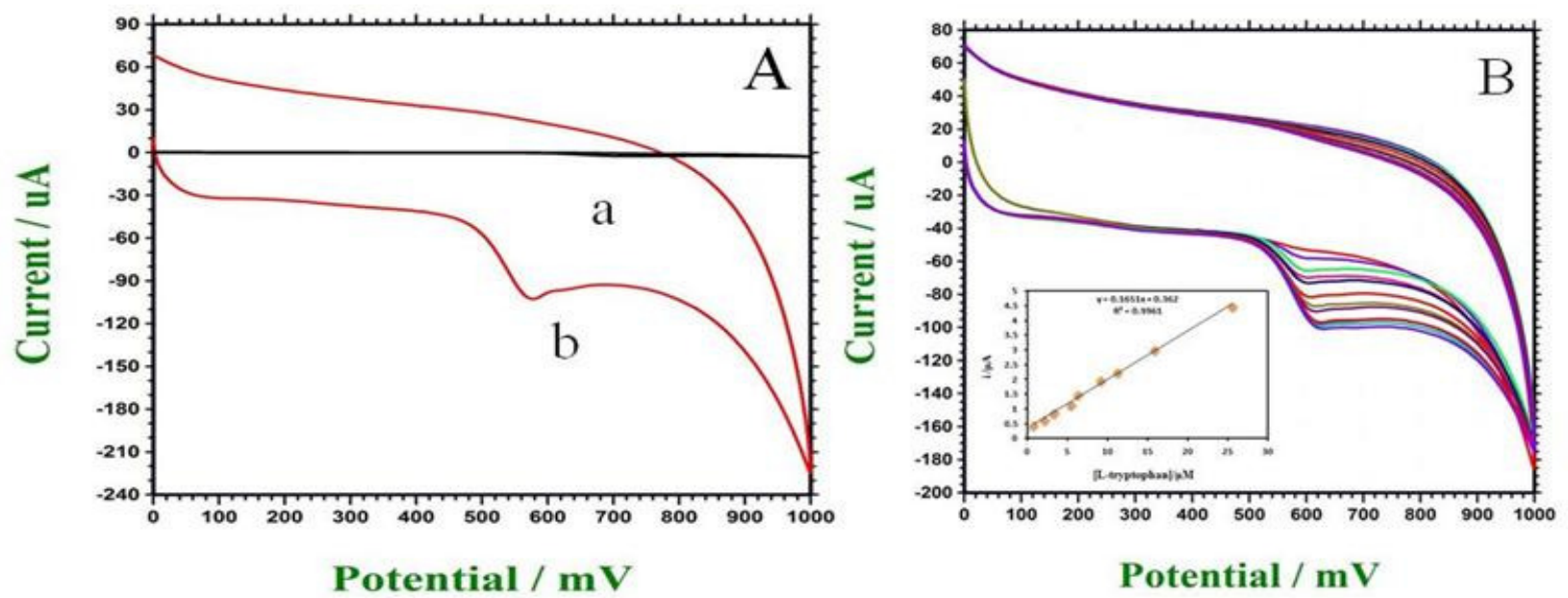

Fig.-(4A). CVs of bare GC electrode (a) and TEG/GCE (b) $10 \mathrm{mM} \mathrm{KCl}$ with $5 \mathrm{mM}$ of $\mathrm{K}_{3}\left[\mathrm{Fe}(\mathrm{CN})_{6}\right]$ at $50 \mathrm{mVS}^{1 .}$, (B) Impedance spectrum of bare and TEG/GCE electrode containing $10 \mathrm{mM} \mathrm{KCl} / 5 \mathrm{mM}$ of $\mathrm{K}_{3}\left[\mathrm{Fe}(\mathrm{CN})_{6}\right]$ at $50 \mathrm{mVS}^{1}$.
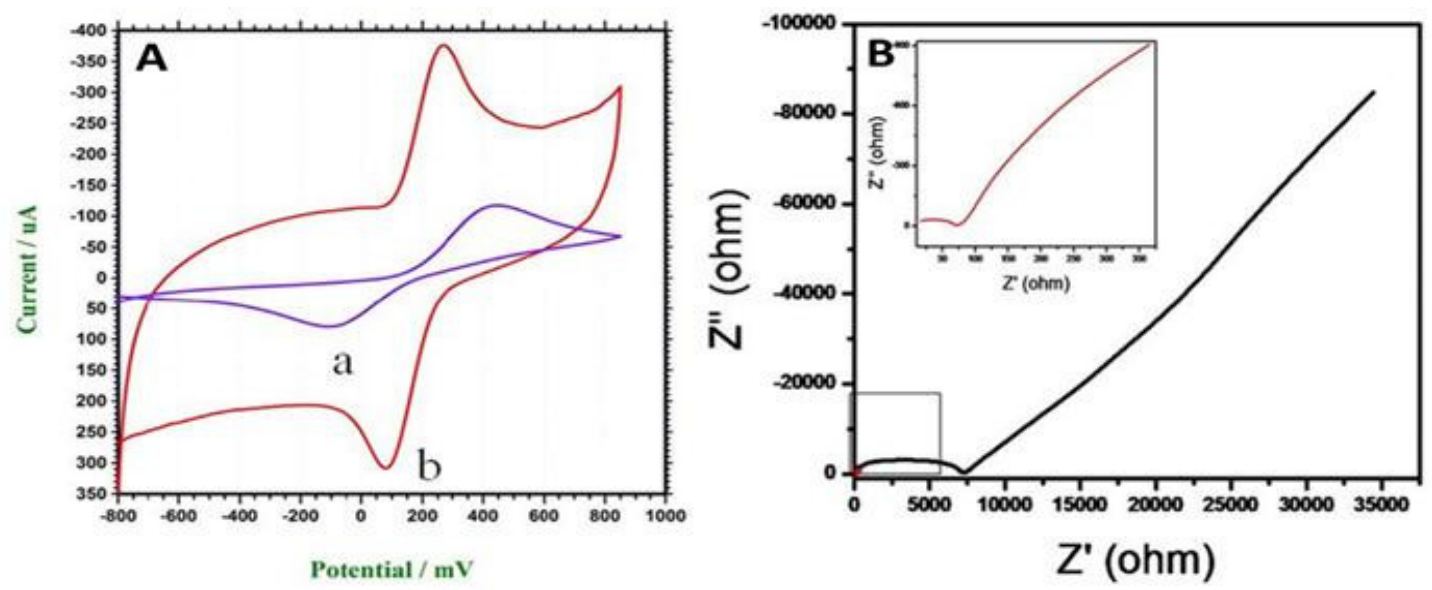

Fig.-5(A) CVs of bare(a) GCE and (b) TEG/GCE modified electrode in presence of L-Trp containing 0.1M Phosphate buffer solution at scan rate $50 \mathrm{mVs}^{-1}$, (B) CVs of TEG/GCE modified electrode containing in various concentration of L-Trp from $0.1 \mathrm{M}$ phosphate buffer solution at scan rate of $50 \mathrm{mVs}^{-1}$. 
The inset in Fig.-6 shows the corresponding calibration plot of catalytic current response versus concentration of $3.2 \mu \mathrm{M}$ to $1 \mathrm{mM}$ for L-Trp shows a linear response of correlation coefficient $\left(\mathrm{R}^{2}=0.9974\right)$. The sensitivity with modified electrode at $0.1464 \mu \mathrm{A} / \mu \mathrm{M}$ and the detection limit $[\mathrm{S} / \mathrm{N}]=3$ ) was establish to be $1.06 \mu \mathrm{M}$. The linear response of L-Trp shows the good electrochemical activity of modified electrode surface involves the electrode, electrolyte interference in the flow system. Evidently, the analytical performances parameter of the proposed sensor is compared with further modified electrodes used in the electrocatalysis of L-Trp are summarized in Table-1.

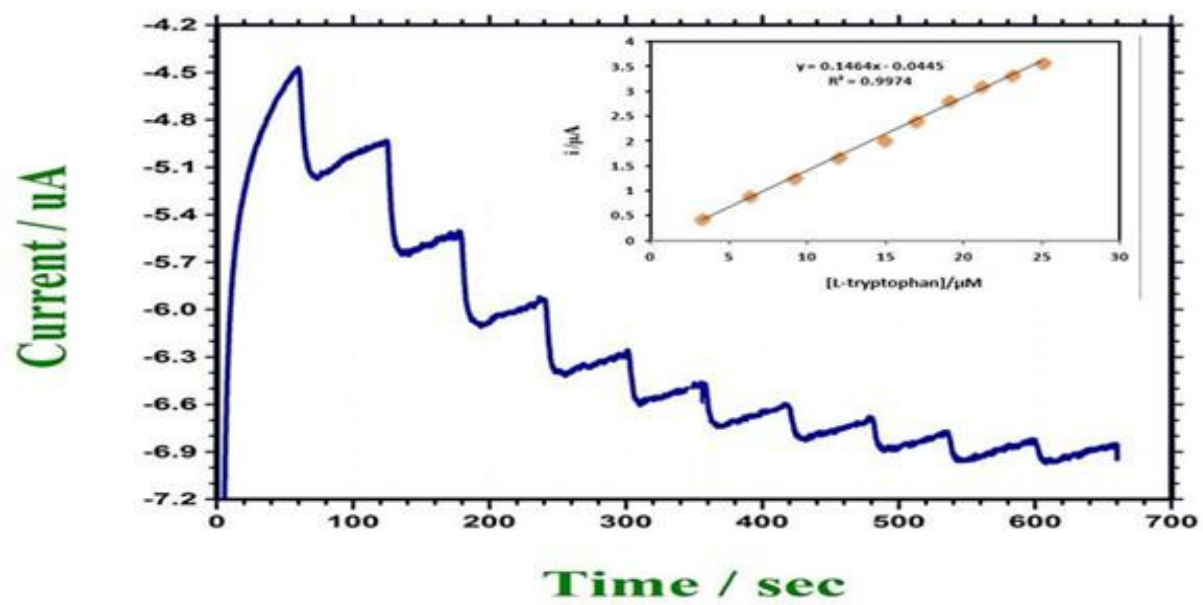

Fig.-6(A)Amperometric of steady-state catalytic current time reaction of the TEG/GCE with a following injection of L-Trp in the dynamic condition in phosphate buffer saline solution, at a fixed potential of $+0.60 \mathrm{~V}$. and (B)Insert the calibration plot of conc. L-Trp Vs peak current.

\section{Differential Pulse Voltammetry}

Differential pulse voltammetry (DPV) is a widely used analytical method for sensitivity and specificity growth in quantitative analysis studies and is compared between predictable CV methods, and the DPV approach uses signals as environmental specificity. ${ }^{22}$

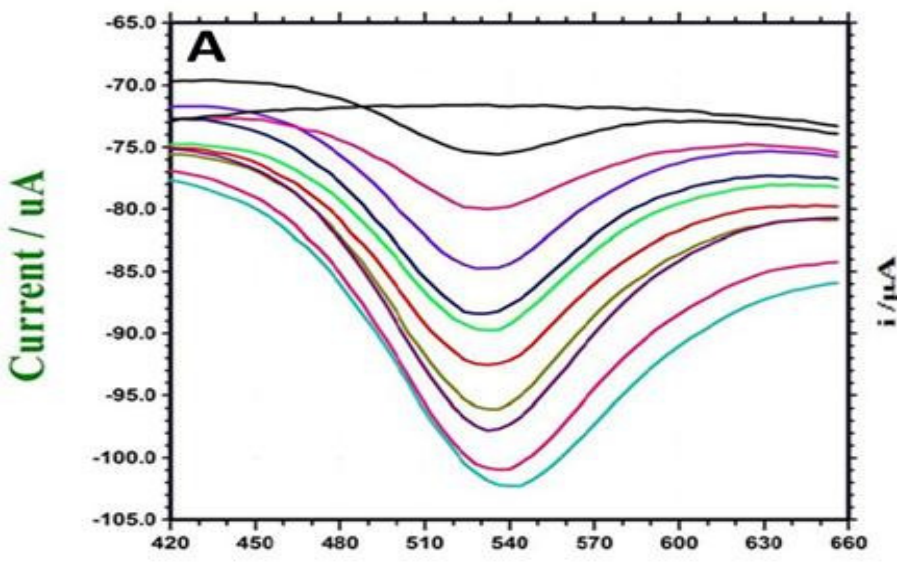

Potential / mV

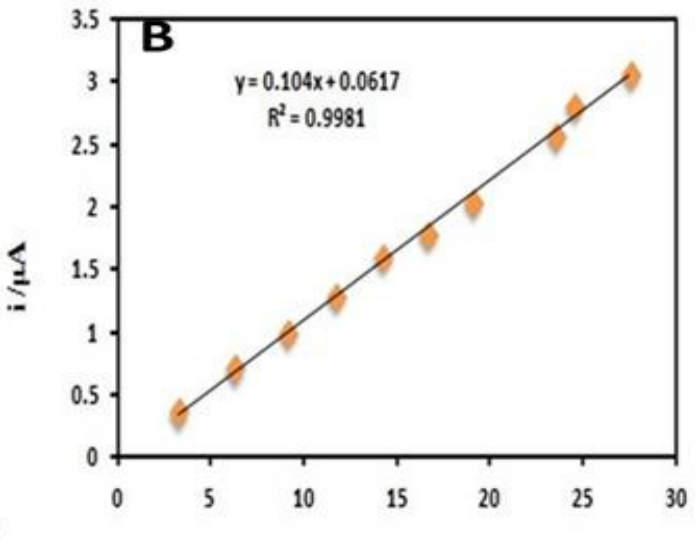

[l-tryptophan]//M

Fig.-7(A) DPV of TEG/GCE modified electrode in addition of L-Trp at the 0.1 M PBS (pH 7.0) above the range of $13 \mu \mathrm{M}$ to $1 \mathrm{mM}$, (B) Insert calibration plot concentration Vs peak current

Figure-7A resolve of the L-Trp was examined by DPV get by scanning the potential series from $0.4 \mathrm{~V}$ to $0.8 \mathrm{~V}$ vs. SCE and the peak current was increased in addition of L-Trp at the 0.1 M PBS (pH 7.0) above the range of $13 \mu \mathrm{M}$ to $1 \mathrm{mM}$. The inset in Fig. 7 show the corresponding calibration plot of catalytic current response against concentration of $3.2 \mu \mathrm{M}$ to $1 \mathrm{mM}$ for L-Trp shows a linear reaction of correlation 
RASĀYAN $J$. Chem.

Vol. 10 | No. 4 |1232-1241 | October - December | 2017

coefficient $\left(\mathrm{R}^{2}=0.9979\right)$. The limit of detection was $1.07 \mu \mathrm{M}$ at a indication to noise percentage of 3 , which was considerably lesser than the previous reports. The lesser detection limit can be certified to the excellent electrocatalytic effect of TEG in L-Trp determination under physiological conditions.

\section{Interruption Studies}

The influence of different substances on the determination of L-Trp $\left(1.0 \times 10^{-4} \mathrm{M}\right)$ was calculated under the nearly all conditions. Cyclic voltammograms of modified electrode for the oxidation of $1.0 \times 10^{-4} \mathrm{M} \mathrm{L-}$ Trp in a presence of $1.0 \times 10^{-3} \mathrm{M} \mathrm{L}$-Tyrosine showed not interfere in the L-Trp peak potential. The peak current of the L-Trp was also not interfered by the addition of $1.0 \times 10^{-3} \mathrm{M}$ uric acid and ascorbic acid, owing to these analytes oxidizes at very low potentials. The highest concentration of interfering amino acids causative of double concentration of L-lysine, L-leucine, L-arginine, L-methionine, L-cysteine, L-valine, L-serine was accurately observed 1.0 The error for determining L-Trp of $\times 10-4 \mathrm{M}$ is less than $5 \%$. In addition, the increase in the concentration of monovalent and divalent ions such as $\mathrm{Na}^{+}, \mathrm{K}^{+}, \mathrm{NH}_{4}{ }^{+}, \mathrm{Mn}^{2+}$, $\mathrm{Zn}^{2+}, \mathrm{Cl}^{-}, \mathrm{Ca}^{2+}, \mathrm{Ba}^{2+}, \mathrm{SO}_{4}{ }^{2-}$ and $\mathrm{NO}_{3}{ }^{-}$did not interfere with Trp measurement.

Table-1: Comparison of L-Trp sensor used in different modified electrodes.

\begin{tabular}{|c|c|c|c|c|c|}
\hline Modified electrodes & Technique & $\begin{array}{c}\text { Linear range } \\
(\mu \mathrm{M})\end{array}$ & $\begin{array}{l}\text { LOD } \\
(\mu \mathrm{M})\end{array}$ & $\begin{array}{c}\text { Oxidation potent } \\
\text { (V) }\end{array}$ & Ref. \\
\hline 4-ABA polymer GCE & $\mathrm{CA}$ & $1.0-100$ & 0.2 & 0.94 & [23] \\
\hline MWCNT-CoSal & DPV & $0.5-50$ & 0.1 & 0.93 & [22] \\
\hline $\mathrm{AgNPs} / \mathrm{CS}-\mathrm{MoS}_{2} / \mathrm{GCE}$ & DPV & $0.5-120$ & 0.05 & 0.93 & {$[24]$} \\
\hline AuNp/GCE & DPV & $0.09-0.5$ & 0.08 & 0.9 & {$[25]$} \\
\hline Hemin/GCE & DPV & $0.1-100$ & 0.025 & 0.88 & [26] \\
\hline Boron-doped diamond & DPV & $20-1000$ & 10 & 0.86 & [27] \\
\hline Nafion/TiO ${ }_{2}$-GR-GCE & DPV & $5-140$ & 0.7 & 0.78 & [28] \\
\hline $\mathrm{CNF}-\mathrm{CPE}$ & $\mathrm{CA}$ & $0.1-119$ & 0.1 & 0.78 & [29] \\
\hline $\mathrm{GR} / \mathrm{Co}_{3} \mathrm{O}_{4} / \mathrm{Nafion} / \mathrm{GCE}$ & SWV & $0.05-10$ & 0.01 & 0.77 & [30] \\
\hline $\mathrm{Cu}-\mathrm{CoHCF}$ graphite & & $10.0-900$ & 6 & 0.7 & [31] \\
\hline Butyrylcholine-GCE & $\mathrm{CV}$ & $2.0-60$ & 0.6 & 0.67 & [32] \\
\hline CuHCF film modified & $\mathrm{CV}$ & $0.85-120$ & 0.018 & 0.65 & {$[14]$} \\
\hline ERGO/GCE & $\mathrm{CV}$ & $0.2-40$ & 0.1 & 0.61 & [33] \\
\hline PCFNiNP modified electrode & $\mathrm{CV}$ & $8.3-1000$ & 2.7 & 0.54 & {$[34]$} \\
\hline TEG/GCE & DPV & $3.2-1000$ & 1.07 & 0.53 & This work \\
\hline
\end{tabular}

\section{Stability of Electrode}

From the amperometric studies were recorded for bare GCE and TEG/GCE were shown in Fig.-8. Chronoamperometry was employed to test the exploit of these two electrodes. The electrode potential was stepped to the oxidation over the potential of L-Trp in both cases. For the TEG/GCE, the steady-state current remained at a higher value than for the bare GCE. This might be the cause for the high catalytic activity of these electrodes. Such as TEG/GCE were having a great surface area and extraordinary catalytic currents having desirable for applications.

\section{Application to Present Sample Analysis}

Towards evaluate the practical utility of the projected TEG modified electrode, the recovery of L-Trp was determined in milk samples. The standard addition method was used for the analysis of the real samples. The data given in Table- 2 shows the suitable result. The recovery of the spiked milk samples was observed to be good for the determinations, suggesting that the TEG modified electrode was really dependable, selective and sensitive for the function of L-Trp in actual samples. 
Table-2: Determination of L-Trp in milk samples $(\mathrm{n}=5)$

\begin{tabular}{c|c|c|c|c}
\hline Sample & \multicolumn{1}{|c|}{ Concentration of L-Trp $(\mu \mathrm{M})$} & Milk sample $(\mu \mathrm{M})$ & Recovery (\%) \\
\hline & Added $(\mu \mathrm{M})$ & Found $(\mu \mathrm{M}) \mathrm{a}$ & & 102.4 \\
\hline 2 & 150.0 & $153.67 \pm 0.12$ & $3.67 \pm 0.12$ & 102.8 \\
\hline & 150.0 & $154.34 \pm 0.16$ & $4.34 \pm 0.16$ & \\
\hline
\end{tabular}

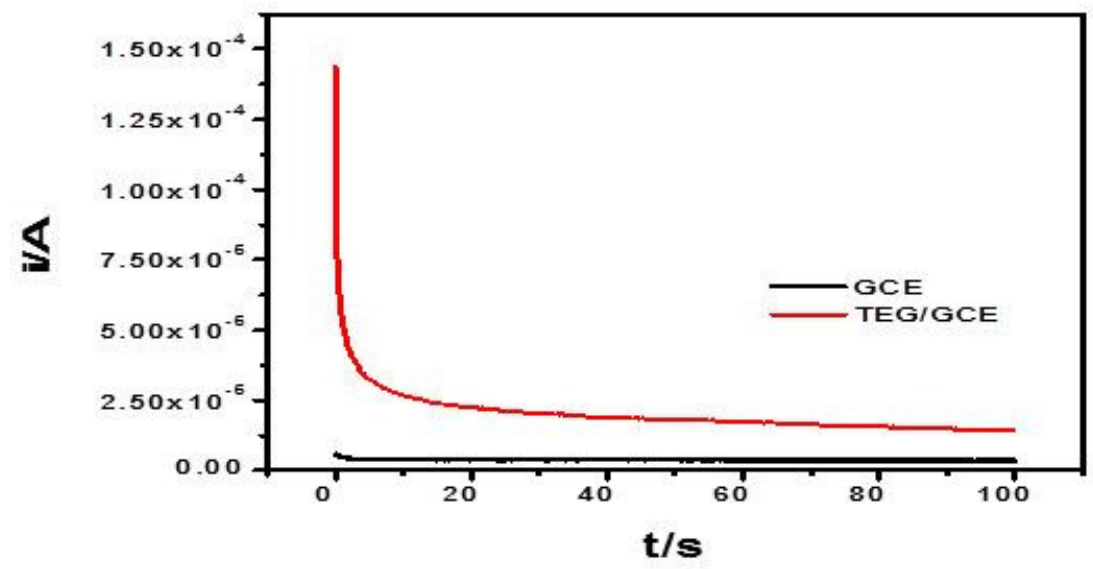

Fig.-8: Amperometric studies of the bare GCE and TEG/GCE with L-Trp in the dynamic condition in phosphate buffer saline solution.

\section{CONCLUSION}

In this work, a novel route for the production of graphene via sulfur intercalated and thermal exfoliation method, followed by graphene was used for the fabrication of modified electrode by drop and dry technique. The electrochemical study was done by modifying TEG/GCE and it process excellent electrocatalytic activity and voltammetry response for the oxidation of the L-Trp. The modified electrode can be detected with large sensitivity and low detection limit and wonderful electrocatalytic presentation can be attributed to the superior biocompatibility and tall conductivity of modified TEG/GCE toward L-Trp. The purpose of the TEG modified electrode for the detection of $\mathrm{L}$ Trp in milk samples show the practical efficacy of the sensor.

\section{ACKNOWLEDGEMENT}

The author would like to acknowledge the financial support from DRDO. Kanpur, Government of India and also Vels University, Chennai, India, for providing infrastructure facilities.

\section{REFERENCES}

1. P.R Wallace Physical Review, 71(9), 622 (1947).

2. A. K. Geim, Science, 324(5934), 1530(2009).

3. A. K., Geim and K. S. Novoselov Nature Materials, 6(3), 183(2007).

4. C. N. R. Rao, A. E, Sood, K. E., Subrahmanyam and A. Govindaraj, Angewandte Chemie International Edition, 48(42), 7752(2009).

5. M. J. Allen, V. C. Tung and R. B. Kaner, Chemical Reviews, 110(1), 132 (2010).

6. R. S. Weatherup, B. C. Bayer, R. Blume, C. Ducati, C. Baehtz, R. Schlogl and S. Hofmann, Nano Letters, 11(10), 4154(2011).

7. D. R. Dreyer, R. S. Ruoff and C. W Bielawski, Angewandte Chemie International Edition, 49(49), 9336(2010).

8. L. Ci, Z. Xu, L. Wang, W. Gao, F.Ding, K. F. Kelly and P. M. Ajayan, Nano Res., 1,116(2008). 
RASĀYAN J. Chem.

Vol. 10 | No. 4 |1232-1241 | October - December | 2017

9. M. H. Gass, U. Bangert, A. L. Bleloch, P. Wang, R. R. Nair and A. K. Geim, Nature Nanotechnology, 3(11), 676 (2008).

10. S.S. Datta, D.R. Strachan, S.M. Khamis, A.T.C. Johnson. Nano Lett, 8, 1912 (2008).

11. M. H. Gass, U. Bangert, A.L. Bleloch, P.Wang, R. R. Nair, \& A. K. Geim, Nature Nanotechnology, 3(11), 676 (2008).

12. J. C Meyer, C. O. Girit, M. F. Crommie and A. Zettl, Nature, 454(7202), 319(2008).

13. Q.Yu, J. Lian, S. Siriponglert, H.Li, Y.P. Chen and S. S. Pei, Applied Physics Letters, 93(11), 113103 (2008).

14. A. A.Ensafi and R. Hajian, Analytica Chimica Acta, 580(2), 236(2006).

15. A. R. Fiorucci and É. T. G. Cavalheiro, Journal of Pharmaceutical and Biomedical Analysis, 28(5), 909(2002)

16. P. Prabhu, R.S. Babu and S.S. Narayanan, Colloids and Surfaces B: Biointerfaces, 87(1), 103-108 (2011).

17. D. C. Marcano,D .V. Kosynkin, J. M. Berlin, A. Sinitskii, Z. Sun, A. Slesarev and J.M. Tour, ACS Nano, 4(8), 4806(2010).

18. S. Stankovich, D. A. Dikin, R. D. Piner, K. A. Kohlhaas, A. Kleinhammes, Y. Jia and R.S.Ruoff, Carbon, 45(7), 1558(2007).

19. D. D. L. Chung,. Journal of Materials Science, 22(12), 4190(1987).

20. F. Tuinstra, and J. Koenig, The Journal of Chemical Physics, 53(3), 1126(1970).

21. K. N. Kudin, B. Ozbas, H. C. Schniepp, R. K. Prud Homme, I. A. Aksay and R. Car, Nano Letters, 8(1), 36(2008).

22. S. Shahrokhian and L. Fotouhi, Sensors and Actuators B: Chemical, 123(2), 942(2007).

23. K. J. Huang, C. X. Xu, W. Z. Xie and W. Wang, Colloids and Surfaces B: Biointerfaces, 74(1), 167(2009).

24. X. Xia, Z. Zheng, Y. Zhang, X. Zhao and C. Wang, Sensors and Actuators B: Chemical, 192, 42 (2014).

25. C. Li, Y. Ya and G. Zhan. Colloids and Surfaces B: Biointerfaces, 76(1), 340(2010).

26. C. G., Nan, C. G. Feng, Z. Z, Li, D. J. Ping, and C. H. Qin, Analytica Chimica Acta, 452(2), 245(2002).

27. G. Zhao, Y. Qi and Y. Tian. Electroanalysis, 18(8), 830(2006).

28. Y. Fan, J. H. Liu, H. T. Lu and Q. Zhang, Microchimica Acta, 173(1-2), 241(2011).

29. X. Tang, Y. Liu, H. Hou and T. You, Talanta, 80(5), 2182(2010).

30. D. Ye, L. Luo, Y. Ding, B. Liu and X. Liu, Analyst, 137(12), 2840(2012).

31. Y. Liu and L. Xu, Sensors, 7(10), 2446(2007).

32. G. P Jin and X. Q. Lin, Electrochemistry Communications, 6(5), 454(2004).

33. K. Q. Deng,, J. H. Zhou and X. F. Li, Colloids and Surfaces B: Biointerfaces, 101, 183(2013).

34. R. S. Babu, P. Prabhu and S. S. Narayanan, Talanta, 110, 135(2013).

[RJC-1749/2017] 